\title{
Optical clarity
}

\section{Are inaccurate or misleading descriptions problems in photonics? Community feedback indicates that there is room for improvement in several areas.}

It may seem obvious that accuracy should be paramount when conveying science, whether the forum is an academic journal or a conference presentation. Accurate communication is just as critical even when the message is aimed at the broader scientific community or the general public. Although we sometimes encounter issues with submitted manuscripts, we at Nature Photonics believe that our contributors generally do a great job at communicating science carefully and accurately.

However, from time to time we receive feedback from the photonics community about some problems in the field regarding the inaccurate communication of science. It will always be possible to find an alternative point of view on a topic, but the feedback is particularly consistent on certain issues. Some of these problem areas are described below.

A commonly raised issue is the determination of electromagnetic mode areas of waveguides. For some simple field distributions, it may be acceptable to use typical determinations such as basic $1 / \mathrm{e}$ definitions for which the mode is considered to be confined within the region where the energy density (or some other relevant parameter) falls to $1 /$ e of its maximum value. This is satisfactory in some simple waveguide structures, but what if the decay of the field from the waveguide varies depending on direction? Furthermore, waveguides with multiple layers can have complicated field distributions. Some layers may be highly dispersive and can hence hold a high energy density; alternatively, there may be large variations in the permittivity between layers, giving rise to sudden changes in the field amplitude. This is especially problematic for very thin layers; in such cases, it is important to properly account for spikes or dips in the field amplitude. These problems are pertinent to many popular modern schemes including dielectric slot waveguides, 'hybrid' plasmonic waveguides and 'dielectric-loaded' plasmonic waveguides. None of the above issues is news: they have been discussed in the literature. Yet field confinements continue to be reported inappropriately in some cases.

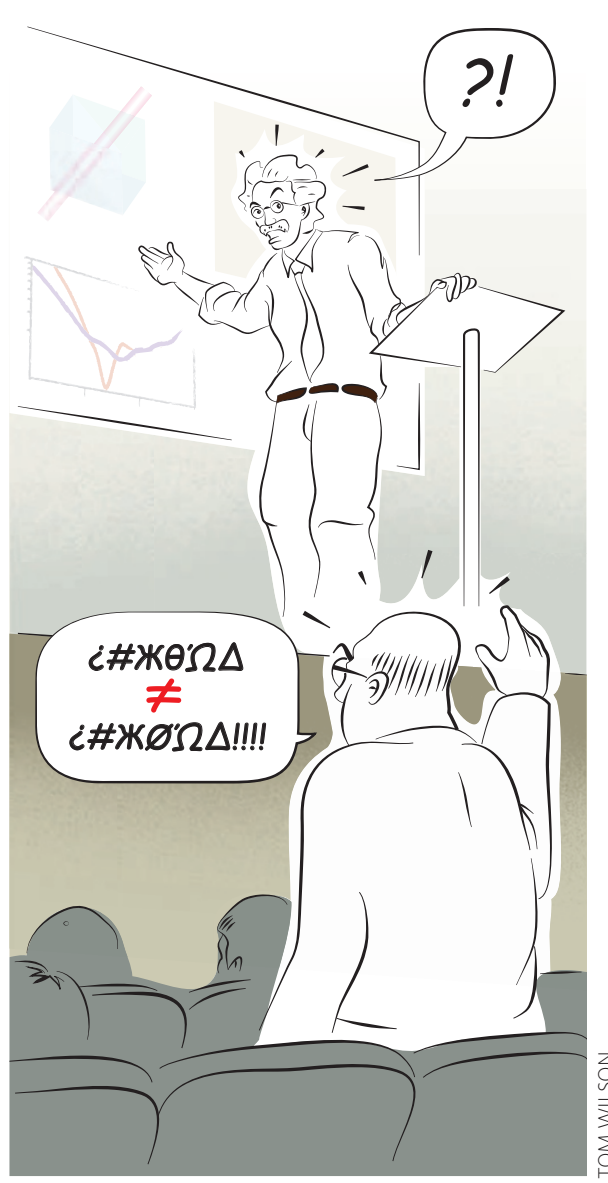

dimensions may be smaller than the field confinement.

In the field of nano-optics, concerns have been raised about the description of imaging approaches that "beat the diffraction limit" or "realize subdiffraction-limit imaging." A valid concern is that such terminology is sometimes used when the results are either barely below the diffraction limit or even slightly above it after appropriately accounting for everything (for example, the numerical aperture and the refractive index).

Several people have questioned whether terahertz plasmonics is indeed plasmonics. This is because at terahertz frequencies metals may contain a very low proportion of the total field energy, so that the unique characteristics typically associated with plasmonics may not be observable in many cases. The observed effects may be better described using other well-established terminology. This is not to say that this direction of research is uninteresting, only that care should be taken with the terminology used to describe it.

Another problematic area is describing simple interference effects (such as the creation of constructive or deconstructive interference regions to form bright (1s) or dark (0s) spots) as active switching. Although interference effects in various structures may be useful, there are several reasons why it is inappropriate to classify well-known interference effects as active optical switching.

Finally, the issue of incorrect claims of breaking reciprocity in reciprocal structures is an ongoing one. It appears to be a persistent misunderstanding of the physical problem, particularly in relation to optical isolation and 'one-way' transmission mirrors. This problem is discussed in more detail in this issue's Commentary on page 579 .

Although some of these issues are just related to using appropriate definitions, it is best to seek to be as accurate as possible, rather than making inaccurate statements, or, as one of our readers expressed it, "to make a good sell of particular research." Naturally, all of us - authors, editors and referees - have our own part to play in ensuring that published science is as accurate as possible. 